\title{
ON THE STRUCTURE OF MAXIMUM MODULUS ALGEBRAS
}

\section{WALTER RUDIN ${ }^{1}$}

Let $U, K$, and $C$ denote the open unit disc, the closed unit disc, and the unit circumference, respectively. In [1], an algebra $R$ of continuous complex functions defined on $K$ was said to be a maximum modulus algebra on $K$ if for every $f \in R$

$$
\max _{z \in K}|f(z)|=\max _{z \in C}|f(z)|
$$

i.e., if every $f \in R$ attains its maximum modulus on $C$. As a matter of convenience, we shall, in this paper, abbreviate "maximum modulus algebra on $K$ " to " $M$-algebra."

Examples of $M$-algebras which come to mind immediately are (a) the algebra $Q$ consisting of all functions which are continuous on $K$ and analytic in $U$, (b) any subalgebra of $a$, (c) any algebra $B$ which is equivalent to an algebra of analytic functions via a homeomorphism of $K$, i.e., any algebra $B$ with which there is associated a homeomorphism $h$ of $K$ into the complex plane, such that every $f \in B$ is of the form

$$
f(z)=f^{*}(h(z))
$$

for some $f^{*}$ which is continuous on $h(K)$ and analytic in $h(U)$.

Does this list contain all $M$-algebras? The results of [1] seemed to point toward a positive answer. In fact, the main theorem of [1], stated in somewhat different form, is as follows:

THEOREM 1. Consider the following two conditions which an $M$ algebra $\mathbb{R}$ may satisfy:

(i) There is a function $h \in R$ which is a homeomorphism of $K$.

(ii) R contains a nonconstant function $\phi \in Q$.

Condition (i) alone implies that $\mathcal{Q}$ is equivalent to an algebra of analytic functions, via $h$. Conditions (i) and (ii) together imply that $R \subset Q$.

The next question that arises naturally is whether (ii) alone implies that $R \subset$ Q. That this is not so was shown by an example in [3]; there an $M$-algebra $\mathbb{R}^{\prime}$ was constructed which was generated by two functions $f$ and $g$, where $f$ was analytic (and not constant) in $U$ and $g$

Presented to the Society February 22, 1958; received by the editors March 5, 1958.

1 The author is a Research Fellow of the Alfred P. Sloan Foundation. 
was not analytic. It is clear, incidentally, that $\mathcal{R}^{\prime}$ cannot be equivalent to any algebra of analytic functions; for if $f(z)=f^{*}(h(z))$ and $g(z)=g^{*}(h(z))$, as in (2), with $f^{*}$ and $g^{*}$ analytic, then the analyticity of $f$ implies that $h$ is analytic (compare $[1, \mathrm{p} .452]$ ), and this forces $g$ to be analytic.

The algebra $R^{\prime}$ does not separate points on $K$ (i.e., there exist $z_{1} \in K, z_{2} \in K$ such that $z_{1} \neq z_{2}$ but $\phi\left(z_{1}\right)=\phi\left(z_{2}\right)$ for every $\left.\phi \in R^{\prime}\right)$. Thus the question arises whether the conclusion " $R \subset Q$ " of Theorem 1 can be rescued if we assume (ii) and some weakened form of (i), for instance, if we replace (i) by the requirement that $R$ should separate points on $K$ (so that there is a canonical homeomorphism of $K$ into the maximal ideal space of the Banach algebra $\bar{R}$, the uniform closure of $R$; we may assume without loss of generality that $R$ contains the constants $[1$, p. 450$])$. The answer, given in the present paper, settles the question raised in [2], and is again negative:

THEOREM 2. There exists a finitely generated $M$-algebra $R$ such that

(a) R separates points on $K$,

(b) $\mathbb{R}$ contains nonconstant functions which are analytic in $U$, and

(c) $R$ contains functions which are not analytic in $U$.

Proof. Let $P$ be a perfect, totally disconnected, bounded subset of the plane, whose two-dimensional Lebesgue measure is positive. Let $Q$ be the set of all points $\left(w_{1}, w_{2}, w_{3}, w_{4}\right)$ in the space of 4 complex variables (i.e., the 8-dimensional euclidean space $E^{8}$ ) such that $w_{i} \in P$ for $i=1,2,3,4 ; Q$ is the cartesian product $P \times P \times P \times P$, embedded in $E^{8}$ in a natural way. Note that both $P$ and $Q$ are homeomorphic to the Cantor set.

There exists a simple closed curve $J$ in the plane such that $P \subset J$. Let $D$ be the interior of $J$. The crux of the proof will be the construction of 4 complex continuous functions $h_{1}, \cdots, h_{4}$ on $K$, with the following properties:

( $\alpha$ ) There exists a subset $H$ of $C$, homeomorphic to the Cantor set, such that the mapping

$$
z \rightarrow\left(h_{1}(z), h_{2}(z), h_{3}(z), h_{4}(z)\right)
$$

is one-to-one on $H$ and maps $H$ onto $Q$.

( $\beta) h_{1} \in A$ and $h_{1}(K-H) \subset D$;

( $\gamma)$ The set $\left\{h_{2}, h_{3}, h_{4}\right\}$ separates points on $K-H$;

( $\delta)$ There is an arc $L \subset U$ on which $h_{2}$ is constant.

(We note that $(\delta$ ) could be replaced by practically any condition which assures nonanalyticity.)

Once we have these functions, we can prove the theorem quite 
rapidly. Since $P$ has positive measure, there exist nonconstant complex functions $q_{1}, q_{2}, q_{3}$ which are continuous in the plane, analytic in the complement of $P$ (including the point at infinity), such that the set $\left\{q_{1}, q_{2}, q_{3}\right\}$ separates points in the plane; for the proof of this, see $[4$, pp. $826-827]$. Let $R$ be the algebra generated by the functions $f_{i j}$, where

$$
f_{i j}(z)=q_{i}\left(h_{j}(z)\right) \quad(i=1,2,3 ; j=1,2,3,4 ; z \in K) .
$$

Condition $(\beta)$ implies that $f_{i 1} \in A$; condition $(\delta)$ implies that $f_{i 2} \notin A$; conditions $(\alpha),(\beta),(\gamma)$ together imply that the set $\left\{h_{1}, h_{2}, h_{3}, h_{4}\right\}$ separates points on $K$, and hence $R$ separates points on $K$. There only remains the verification that $R$ is an $M$-algebra.

Every member of $R$ is of the form

$$
f(z)=g\left(f_{i j}(z)\right)=g\left(q_{i}\left(h_{j}(z)\right)\right),
$$

where $g$ is a polynomial in 12 variables. Put

$$
\phi\left(w_{1}, w_{2}, w_{3}, w_{4}\right)=g\left(q_{i}\left(w_{j}\right)\right) .
$$

If we keep $w_{2}, w_{3}, w_{4}$ fixed, then $\phi$, as a function of $w_{1}$, is analytic in the complement of $P$. The maximum modulus theorem therefore implies that there is a point $w_{1}^{*} \in P$ such that

$$
\left|\phi\left(w_{1}, w_{2}, w_{3}, w_{4}\right)\right| \leqq\left|\phi\left(w_{1}^{*}, w_{2}, w_{3}, w_{4}\right)\right| .
$$

Keeping $w_{1}^{*}, w_{3}, w_{4}$ fixed, and then repeating this procedure twice more, we find that there is a point $\left(w_{1}^{*}, w_{2}^{*}, w_{3}^{*}, w_{4}^{*}\right) \in Q$ such that

$$
\left|\phi\left(w_{1}, w_{2}, w_{3}, w_{4}\right)\right| \leqq\left|\phi\left(w_{1}^{*}, w_{2}^{*}, w_{3}^{*}, w_{4}^{*}\right)\right|
$$

for all $\left(w_{1}, w_{2}, w_{3}, w_{4}\right)$. By $(\alpha)$ there is a point $z^{*} \in H$ such that $h_{j}\left(z^{*}\right)$ $=w_{j}^{*}(j=1, \cdots, 4)$, and a glance at (4), (5), and (7) shows that

$$
|f(z)| \leqq\left|f\left(z^{*}\right)\right|
$$

for all $z \in K$.

Thus $R$ is an $M$-algebra, and Theorem 2 follows.

We now turn to the construction of the functions $h_{1}, \cdots, h_{4}$ and of the set $H$.

Let $E$ be a perfect subset of $C$, of measure zero. There exist complex continuous functions $\phi_{1}, \cdots, \phi_{4}$, defined on $E$, such that the mapping

$$
t \rightarrow\left(\phi_{1}(t), \phi_{2}(t), \phi_{3}(t), \phi_{4}(t)\right)
$$

is a homeomorphism of $E$ onto $Q$. By the theorem proved in [3], there exists a function $f_{1} \in A$, such that $f_{1}(t)=\phi_{1}(t)$ for all $t \in E$ and 
such that $f(K) \subset D \cup J$. Let $K^{\prime}$ be the closed convex hull of $E$, let $\psi$ be a conformal map of $K$ onto $K^{\prime}$ (i.e., $\psi$ is a homeomorphism of $K$ onto $K^{\prime}$ which is conformal in the interior of $K$ ), and put $H=\psi^{-1}(E)$. Define

$$
h_{1}(z)=f_{1}(\psi(z)) \quad(z \in K),
$$

and

$$
h_{j}(z)=\phi_{j}(\psi(z)) \quad(j=2,3,4 ; z \in H) .
$$

Then condition $(\beta)$ holds, and if we can extend $h_{2}, h_{3}, h_{4}$ from $H$ to $K$ so that $(\gamma)$ and $(\delta)$ are satisfied, the proof will be complete, since $(\alpha)$ is implied by our choice of $\left\{\phi_{j}\right\}$.

Triangulate $K-H$; each compact subset of $K-H$ will be covered by a finite collection of triangles (some of these will be curvilinear), and every point of $H$ will be a limit point of the set $T$ of vertices. Pick two vertices $t^{\prime}, t^{\prime \prime} \in U$ which are joined by an edge of our triangulation, and define $h_{j}(t)$ for $j=2,3,4$ and $t \in T$ such that $h_{j}$ is continuous on $H \cup T$, such that

$$
h_{2}\left(t^{\prime}\right)=h_{2}\left(t^{\prime \prime}\right)=0,
$$

and such that the points $h(t)=\left(h_{2}(t), h_{3}(t), h_{4}(t)\right)$ are in general position in $E^{6}$; i.e., no $m+2$ of these points lie in any linear $m$-space, for $m=1, \cdots, 4$.

Let $\Delta$ be one of our triangles, with vertices $t_{1}, t_{2}, t_{3}$. Define $h_{j}(z)$ for $z \in \Delta$ so that the mapping

$$
z \rightarrow\left(h_{2}(z), h_{3}(z), h_{4}(z)\right)
$$

is a homeomorphism of $\Delta$ onto the (rectilinear) triangle whose vertices are the points $h\left(t_{1}\right), h\left(t_{2}\right), h\left(t_{3}\right)$ in $E^{6}$.

The functions $h_{j}$ are now extended to $K$ and are continuous on $K$.

Since the points $h(t)$ are in general position, no two triangles whose vertices are among these points will intersect, except possibly in a common vertex or a common edge. It follows that condition $(\gamma)$ is satisfied; and (12) shows that condition $(\delta)$ also holds, with the interval $\left[t^{\prime}, t^{\prime \prime}\right]$ for $L$.

This completes the proof of the theorem. It seems quite likely that another proof can be given by exhibiting an example with fewer generators; their number can perhaps be pushed down to 2 , but different methods are needed for this.

In conclusion, we pose another problem:

Suppose $R$ is an $M$-algebra such that $Q \cap R$ separates points on $K$. Does it follow that $R \subset Q$ ? 


\section{REFERENCES}

1. Walter Rudin, Analyticity and the maximum modulus principle, Duke Math. J. vol. 20 (1953) pp. 449-458.

2. - Research Problem 19, Bull. Amer. Math. Soc. vol. 60 (1954) p. 399.

3. - Boundary values of continuous analytic functions, Proc. Amer. Math. Soc. vol. 7 (1956) pp. 808-811.

4. - Subalgebras of spaces of continuous functions, Proc. Amer. Math. Soc. vol. 7 (1956) pp. 825-830.

UNIVERSITY OF ROCHESTER

\section{ON A CLASS OF UNIVERSAL ORDERED SETS}

\section{ELLIOTT MENDELSON}

An ordered set $B$ is said to be $\boldsymbol{\aleph}_{\alpha}$-universal if and only if every ordered set of power $\boldsymbol{\aleph}_{\alpha}$ is similar to a subset of $B$. Let $U_{\omega_{\alpha}}$ be the lexicographically ordered set of all sequences of 0 's and 1's of type $\omega_{\alpha}$; and let $H_{\alpha}$ be the subset of $U_{\omega_{\alpha}}$ consisting of all sequences $\left\{x_{\xi}\right\}_{\xi<\omega_{\alpha}}$ for which there is some $\xi_{0}<\omega_{\alpha}$ such that $x_{\xi_{0}}=1$ and, for $\xi>\xi_{0}, x_{\xi}=0$.

$H_{0}$, being countable, dense, and without first or last element, is similar to the set of rationals in their natural order, and therefore, is $\boldsymbol{\aleph}_{0}$-universal. Sierpiński [2] has shown (as a direct consequence of his theorem that $H_{\alpha+1}$ is an $\eta_{\alpha+1^{-}}$-set) that, for any $\alpha, H_{\alpha+1}$ is $\boldsymbol{\aleph}_{\alpha+1^{-}}$ universal. Gillman [1] has given a demonstration that, for any limit ordinal $\alpha, H_{\alpha}$ is $\boldsymbol{\aleph}_{\alpha}$-universal. The purpose of this note is to give a very simple proof of these results, which does not depend on the ordinal $\alpha$.

TheOREM. $H_{\alpha}$ is $\boldsymbol{\aleph}_{\alpha}$-universal.

Proof. Let $A$ be an ordered set of power $\boldsymbol{\aleph}_{\alpha}$. Fix some well-ordering $\left\{a_{\beta}\right\}_{\beta<\omega_{\alpha}}$ of $A$. Let $\prec$ denote the order in $A$. Define a function $\phi$ from $A$ into $H_{\alpha}$ in the following way. Let $a_{\tau}$ be an element of $A$, and $\beta<\omega_{\alpha}$. Then the $\beta$ th component $\phi_{\beta}\left(a_{\tau}\right)$ of $\phi\left(a_{\tau}\right)$ is defined by:

$$
\phi_{\beta}\left(a_{\tau}\right)= \begin{cases}1 & \text { if } \beta \leqq \tau \text { and } a_{\beta} \leqq a_{\tau}, \\ 0 & \text { otherwise. }\end{cases}
$$

Now, let $a_{\tau}$ and $a_{\sigma}$ be any elements of $A$, with $a_{\tau}<a_{\sigma}$. Clearly, if $\beta \leqq \sigma, \phi_{\beta}\left(a_{\sigma}\right) \geqq \phi_{\beta}\left(a_{\tau}\right)$. But, $\phi_{\sigma}\left(a_{\sigma}\right)=1$ and $\phi_{\sigma}\left(a_{\tau}\right)=0$. Hence, $\phi\left(a_{\tau}\right)$ pre-

Received by the editors March 27, 1958. 